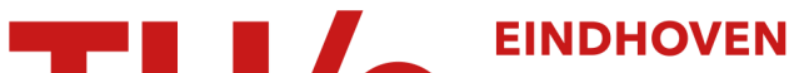 UNIVERSITY OF TECHNOLOGY
}

\section{Finite-difference Green's function diakoptics : unlimited stable lookback schemes for non-trivial problems}

\section{Citation for published version (APA):}

Hon, de, B. P., \& Arnold, J. M. (2012). Finite-difference Green's function diakoptics : unlimited stable lookback schemes for non-trivial problems. In Proceedings of the 2012 International Conference on Electromagnetics in Advanced Applications (ICEAA '12), September 2-7, 2012, Cape Town, South Africa (pp. 926-929). Institute of Electrical and Electronics Engineers. https://doi.org/10.1109/ICEAA.2012.6328767

DOI:

10.1109/ICEAA.2012.6328767

Document status and date:

Published: 01/01/2012

\section{Document Version:}

Accepted manuscript including changes made at the peer-review stage

\section{Please check the document version of this publication:}

- A submitted manuscript is the version of the article upon submission and before peer-review. There can be important differences between the submitted version and the official published version of record. People interested in the research are advised to contact the author for the final version of the publication, or visit the $\mathrm{DOI}$ to the publisher's website.

- The final author version and the galley proof are versions of the publication after peer review.

- The final published version features the final layout of the paper including the volume, issue and page numbers.

Link to publication

\section{General rights}

Copyright and moral rights for the publications made accessible in the public portal are retained by the authors and/or other copyright owners and it is a condition of accessing publications that users recognise and abide by the legal requirements associated with these rights.

- Users may download and print one copy of any publication from the public portal for the purpose of private study or research.

- You may not further distribute the material or use it for any profit-making activity or commercial gain

- You may freely distribute the URL identifying the publication in the public portal.

If the publication is distributed under the terms of Article 25fa of the Dutch Copyright Act, indicated by the "Taverne" license above, please follow below link for the End User Agreement:

www.tue.nl/taverne

Take down policy

If you believe that this document breaches copyright please contact us at:

openaccess@tue.nl

providing details and we will investigate your claim. 


\title{
Finite-difference Green's function diakoptics - Unlimited stable lookback schemes for non-trivial problems
}

\author{
B. P. de Hon ${ }^{1} \quad$ J. M. Arnold ${ }^{2}$
}

\begin{abstract}
Earlier, we have analysed a finite-difference timedomain (FDTD) toy problem for a configuration with two disjoint domains, each consisting of a single point, using a Green's function diakoptics unlimited lookback scheme, based on the inverse of the stability function.

We have developed an analogous scheme for non-trivial problems involving at least one interior point in one of the domains that occurs in the stability function, but does not enter directly into the boundary operator of the lookback scheme. This scheme preserves the stability. If self-consistency regarding the use of the proper discrete Green's functions is maintained, the boundary operator remains restricted to the boundary. Otherwise the boundary operator fills up to become a domain operator, but may still be usable.
\end{abstract}

\section{INTRODUCTION}

In order to address space-time field problems involving wave interaction between disjoint domains, we have previously reported on finite-difference Green's function diakoptics (GFD). The GFD scheme in [1] is a self-consistent finite lookback scheme derived from a $Z$-domain representation for a boundary operator that describes the wave interaction between the computational domains and the unbounded environment. With this scheme stability is guaranteed, but spurious recurrences may only be suppressed through the introduction of small losses, which is unacceptable for describing the wave interaction between distant domains. In [2], we considered a toy problem for two domains, each consisting of a single point, for which we proposed an unlimited lookback scheme based on the inverse of the stability function. In this robust scheme, stability is guaranteed, while the spurious recurrences are kept at bay. Even if one chooses to sacrifice self-consistency and replaces the inter-domain FDTD Green's functions by continuous space-time Green's functions, the stability of the unlimited lookback scheme is still retained.

However, the toy problem is trivial in that the two points automatically coincide with the boundaries of the respective domains. In a non-trivial problem there is at least one interior point in one of the domains that occurs in the stability function, but does not enter directly into the boundary operator. We shall derive an unlimited lookback scheme for a non-trival GFD problem, and in- vestigate the ramifications of partially interfering with the discrete Green's function operator.

\section{NON-TRIVIAL DIAKOPTICS}

Let us consider an FDTD wavefield $\phi$ on a $\nu$ dimensional hypercubic lattice with unit grid size, as it is excited in discrete time by a source distribution $q$. For simplicity we adjust the time-step to the grid size, such that the Courant number $\alpha=1$ [3]. The associated time sequence at grid-point $k \in \mathbb{Z}^{v}$ is denoted as $\phi_{k}^{n}$ with $n \in \mathbb{Z}$. Further, we assume that the sources start to act at $n=0$, and employ $Z$-transformed quantities, e.g., $\phi_{k}(Z)=\sum_{n=0}^{\infty} \phi_{k}^{n} Z^{-n}$. In our diakoptics approach, our computational domain $\mathcal{D}$ is the union of disjoint bounded subdomains of $\mathbb{Z}^{v}$. Its complement $\overline{\mathcal{D}}$ is unbounded. Further, we define the sets of interior and exterior boundary points $\partial \mathcal{D}_{\mathrm{i}}$ and $\partial \mathcal{D}_{\mathrm{e}}$ as those points in $\mathcal{D}$ and $\overline{\mathcal{D}}$ that are adjacent to $\overline{\mathcal{D}}$ and $\mathcal{D}$, respectively.

With reference to [4], we may cast the $Z$-domain counterpart of the diakopted FDTD scheme on $\mathcal{D}$ in the following form

$$
\mathrm{S} \phi=\mathbf{q} \text { with } \mathrm{S}=\mathrm{P}+\mathrm{F}-\zeta \mathrm{I}
$$

where $\boldsymbol{\phi}$ and $\mathbf{q}$ denote the respective $N$-dimensional vector representations of the field and source distributions, $\mathrm{P}$ represents the boundary operator that incorporates the field history on $\partial \mathcal{D}_{\mathrm{i}}$ into the FDTD update equations, while $\mathrm{F}, \mathrm{I}$, and $\mathrm{S}$, are the negative mesh Laplacian restricted to $\mathcal{D}$, and the identity and stability operators, respectively. In (1), we have also introduced $\zeta=v\left(2-Z-Z^{-1}\right)$.

In [2], we considered a toy problem for which the disjoint domains consist of single points, located at $k_{0}$ and $k_{1}$, and introduced an unlimited lookback scheme by which the boundary operator may be constructed on the fly. Let the Green's functions $G_{0}$ and $G_{k}$ denote the fields at $k_{0}\left(k_{1}\right)$ and $k_{1}\left(k_{0}\right)$, respectively, due to a unit-amplitude sources at $k_{0}\left(k_{1}\right)$. Formally inverting (1) gives $\phi=(S)^{-1} \mathbf{q}$, where the inverse of the stability

\footnotetext{
${ }^{1}$ Eindhoven University of Technology, Department of Electrical Engineering, P.O.Box 513, 5600 MB Eindhoven, The Netherlands, e-mail: B.P.d.Hon@tue.nl, tel.: +31 402473603 , fax: +31402448375 .

${ }^{2}$ School of Engineering, University of Glasgow, Glasgow G12 8QQ, United Kingdom, e-mail: John. Arnoldeglasgow. ac . uk, tel.: +44141330 5217, fax: +441413304907.
} 
operator follows by inspection as

$$
(\mathrm{S})^{-1}=\left(\begin{array}{cc}
G_{0} & G_{k} \\
G_{k} & G_{0}
\end{array}\right)
$$

If $\operatorname{det}(\mathrm{S})$ has no zeroes outside the unit circle in the complex $Z$-plane, the stability is guaranteed, and vice versa. Since the $Z$-domain Green's functions and hence $\mathrm{S}^{-1}$ exist for any $Z$ with $|Z|>1$, we infer that $\operatorname{det}(\mathrm{S})$ cannot vanish for $|Z|>1$, and hence, that stability is guaranteed as long as we do not introduce spurious zeroes outside $|Z|=1$ in the process of transforming back to the discrete time domain. For this toy problem, the special structure of $\mathrm{S}^{-1}$ can be exploited to derive a coupled system of recurrence schemes for the two time sequences $S_{11}^{n}=S_{22}^{n}$ and $S_{12}^{n}=S_{22}^{n}$, for $n=-1,0,1,2, \ldots$.

Let us follow an analogous procedure for generic non-trivial problems, especially, problems with internal points, i.e., points in $\mathcal{D}$ that do not lie on $\partial \mathcal{D}_{\text {i. }}$. Let

$$
(\mathrm{S})^{-1}=\mathrm{G}=\sum_{n=1}^{\infty} Z^{-n} \mathrm{G}^{n},
$$

where $G$ is the matrix of Green's functions describing the interaction between all points in $\mathcal{D}$, which is symmetric on account of reciprocity, and $\mathrm{G}^{n}$ are the corresponding times sequences. The delay in the FDTD field response to the action of a source implies that $\mathrm{G}=O\left(Z^{-1}\right)$, or equivalently, the corresponding $Z$ transform series starts at $n=1$ for which we find $\mathrm{G}^{1}=(1 / v) \mathrm{I}$. Since $(\mathrm{S})^{-1}=\mathrm{G}$, we have $\mathrm{S}=O(Z)$, and we may formally write

$$
\mathrm{S}=\sum_{n=-1}^{\infty} Z^{-n} \mathrm{~S}^{n}
$$

By definition, $S G=I$, (or $G S=1$ ) which after expansion in powers of $Z^{-1}$ leads to

$$
\begin{aligned}
\mathrm{S}^{-1} & =v \mathrm{l} \\
\mathrm{S}^{n} & =-v \sum_{m=0}^{n} \mathrm{~S}^{n-1-m} \mathrm{G}^{m+2},
\end{aligned}
$$

for $n=0,1, \ldots$. Up to a trivial shift of the indices, we have performed Cauchy product of the sequences $\left\{\mathrm{G}^{n}\right\}$ and $\left\{S^{n}\right\}$, which leads to a matrix recurrence scheme by which $S^{n}$ can be determined. In particular, we have $S^{0}=F-2 v l$, which, in view of $G^{1}=(1 / v) \mid$ and $\mathrm{P}=\mathrm{S}-\mathrm{F}+\zeta \mathrm{I}$ implies that

$$
\begin{aligned}
\mathrm{P}^{-1} & =\mathrm{O}, \\
\mathrm{P}^{0} & =\mathrm{O}, \\
\mathrm{P}^{1} & =-v \mid+\mathrm{S}^{1}, \\
\mathrm{P}^{n} & =\mathrm{S}^{n},
\end{aligned}
$$

for $n=2,3, \ldots$. Hence the remaining successive coefficients in $\mathrm{P}=\sum_{n=1}^{\infty} Z^{-n} \mathrm{P}^{n}$ are associated with proper successive time delays, and form the basis for an unlimited lookback scheme for the field $\phi$ in $\mathcal{D}$.

\section{NUMERICAL RESULTS}

Let us put this procedure to the test. In Figure 1, we have depicted the minimum non-trivial configuration, consisting of two disjoint domains with $N_{\mathcal{D}}=6$ points, i.e., $N_{\mathrm{i}}=5$ interior boundary points (labeled 1 to 5 , encircled), and a single internal point (labeled 6 in the grey circle). The $N_{\mathrm{e}}=12$ exterior boundary points are labeled 1 to 12 inside squares, and the connections between interior and exterior boundary points are labeled 1 to $L=16$.

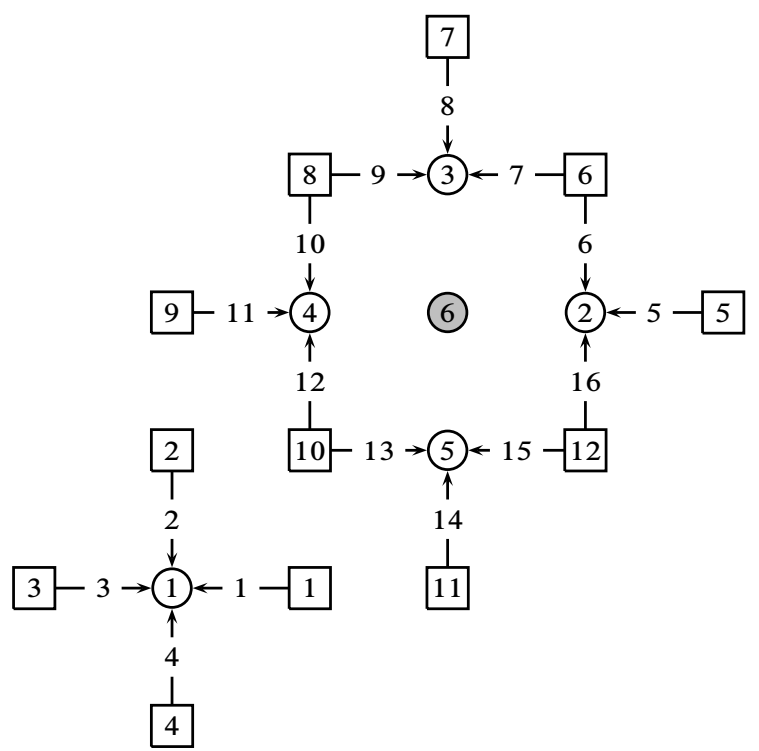

Figure 1: The minimum non-trivial configuration, consisting of two disjoint domains, with five interior boundary points (encircled) a single internal point (grey circle), twelve exterior boundary points (squares), and sixteen connections between interior and exterior boundary points.

In all examples, a source is located in interior boundary point 1 . In our first examples the relative displacement between interior boundary point 1 and internal point 6 is $(400,300)$. We shall display impulse responses $\left(q_{1}^{0}=1, q_{k}^{n}=0\right.$ otherwise) and the corresponding fields due to a source signature, i.e., a Ricker wavelet, with a trough-to-trough separation of about 41.6. The wavelet has been scaled by a constant to render the impulse responses and the fields commensurate.

In the first example, we have used the unlimited lookback scheme to compute the fields in domain $\mathcal{D}$. In Figure 2, we have displayed the results at interior boundary point 1 (the source location). Unsurprisingly, these results would have been exactly the same, if we had directly used the Green's function sequence $\left\{G_{0}^{n}\right\}$, 
and convolution with a Ricker pulse, instead of the unlimited lookback scheme.
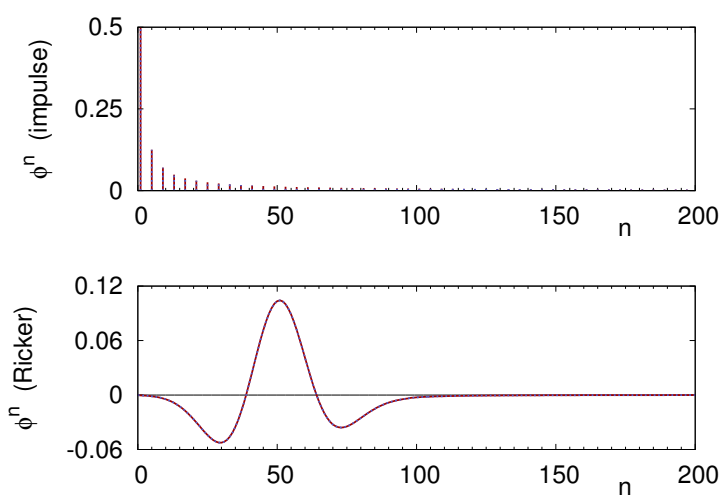

Figure 2: Impulse response at interior boundary point 1 (the source location), and the corresponding field for a Ricker wavelet with troughto-trough separation of about 41.6 , computed using the unlimited lookback scheme. The relative displacement between interior boundary point 1 and internal point 6 is $(400,300)$.

However, working with a non-trivial domain provides us with the opportunity of modifying the properties of the internal part of $\mathcal{D}$ without disturbing the boundary operator itself, which describes the influence of the environment.

In particular, if we replace the internal point, labeled 6 , by a perfect electric conductor (PEC), then scattering off that PEC will occur. Figure 3 shows a latertime window of the incident (blue, dotted) and total field (red, solid) at interior boundary point 1 (the source location). Note that diakoptics automatically takes care of the signal transfer from the source sub-domain to the PEC one and back.
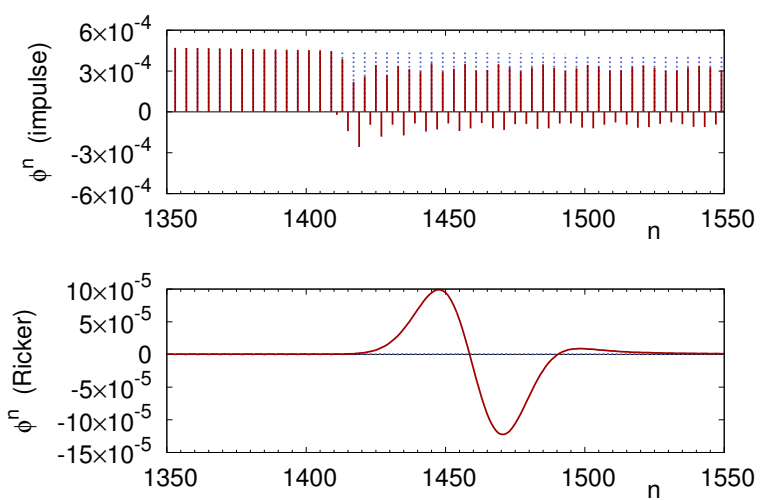

Figure 3: Impulse response at interior boundary point 1 (the source location), and the corresponding field for a Ricker wavelet with troughto-trough separation of about 41.6, computed using the unlimited lookback scheme. The relative displacement between interior boundary point 1 and internal point 6 is $(400,300)$.

Figure 4 shows the incident (blue, dotted) and total field (red, solid) at interior boundary point 3 (just above the PEC).
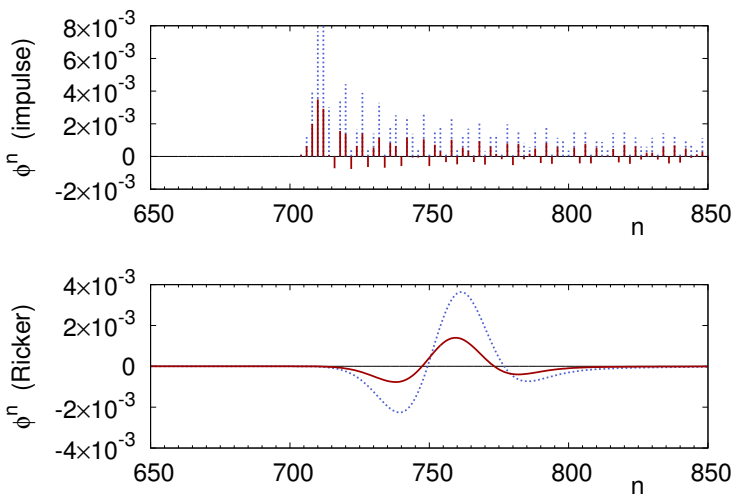

Figure 4: Impulse response at interior boundary point 3, and the corresponding field for a Ricker wavelet with trough-to-trough separation of about 41.6, computed using the unlimited lookback scheme. The relative displacement between interior boundary point 1 and internal point 6 is $(400,300)$.

The results above are indistinguishable from those that could have been compted by running the FDTD evolution on a large grid enveloping both disjoint domains.

The existence of a bounded $S^{-1}$ implies that the unlimited lookback scheme is stable. The discrete Green's functions in (3) guarantee that the resulting scheme is self-consistent, in that it is equivalent to the FDTD scheme in $\mathbb{Z}^{n}$ as far as the points in $\mathcal{D}$ are concerned. As a consequence of the self-consistency, the matrix elements of $\mathrm{P}^{n}$ that correspond to internal points should vanish identically, and this can be exploited in speeding up the evaluation of $\mathrm{S}^{n}$ for $n>1$.

One may choose to replace some of the Green's functions in (3) by alternative functions of $Z$ that are bounded for $Z>1-$ e.g., discretised versions of the continuous Green's function could be used to mitigate long-distance numerical dispersion effects - without affecting the stability (which is crucial). However, this does compromise self-consistency, and as a consequence, the matrix elements of $\mathrm{P}^{n}$ that correspond to internal points no longer vanish.

For Figure 5, we return to the free-space lattice case (no PEC), but replace the discrete Green's functions from interior boundary point 1 to interior boundary points 2, 3, 4, and 5, by the single discrete Green's function from interior boundary points 1 to interior boundary point 3 , while retaining the other discrete Green's function matrix elements. The relative displacement between interior boundary point 1 and internal point 6 is now $(40,30)$.

The figure shows the field at at interior boundary point 3 , computed in three different ways, i.e., in a selfconsistent fashion with proper discrete Green's functions (blue, dotted, but obscured), in an approximate fashion with the single interior boundary point discrete Green's function from point 1 to point 3 (green, dashed), and using the same approximate fashion, followed by the post-computation removal matrix elements of $\mathrm{P}$ associated with the internal points (red, 
solid).
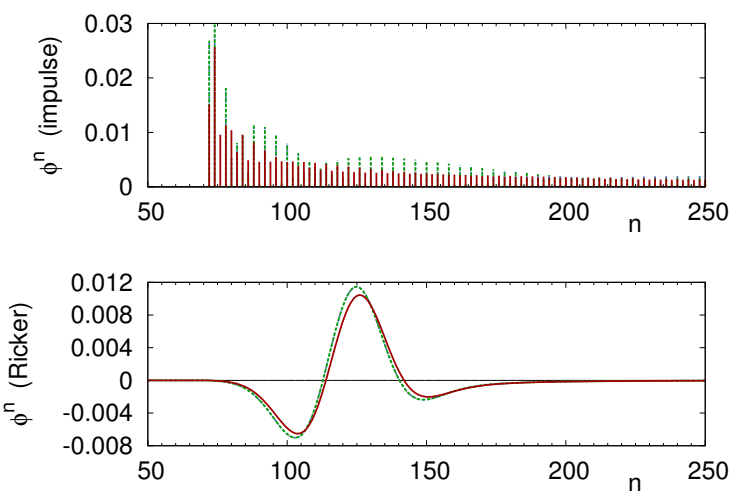

Figure 5: Impulse response at interior boundary point 3, and the corresponding field for a Ricker wavelet with trough-to-trough separation of about 41.6, computed using the unlimited lookback scheme. The relative displacement between interior boundary point 1 and internal point 6 is $(40,30)$.

The full P-matrix altered Green's function approximation overlaps the exact case, indicating that that approximation is quite good. However, the matrix elements associated with the internal points are quite substantial and fior this inter-domain displacement leads for visibly different results.

\section{DISCUSSION}

We have presented an unlimited lookback scheme involving a discrete Green's function diakoptics boundary operator for non-trivial problems. The scheme preserves the stability of the underlying FDTD problem on an infinite grid. In the self-consistent case, involving the proper discrete Green's functions, diakoptics produces the same results as if the grid were infinite. The calculation of the boundary operator involves multiplications of $N_{\mathcal{D}} \times N_{\mathcal{D}}$ matrices, which for the self-consistent case can be reduced to multiplications of $N_{\mathrm{i}} \times N_{\mathrm{i}}$ matrices. If self-consistency is abandoned one must retain all $N_{\mathcal{D}}^{2}$ elements produced by the recurrence scheme for $\left\{\mathrm{P}^{n}\right\}$, implying that $\mathrm{P}$ becomes a domain operator rather than a boundary one.

We shall report on an alternative formulation for $\mathrm{P}$ involving matrices of sizes up to $N_{\mathrm{e}} \times N_{\mathrm{e}}$ elsewhere. The self-consistent scheme relies on the availability of a fast scheme for computing the discrete Green's function time sequences, which for the 2-D case will appear elsewhere as well.

\section{References}

[1] B. P. de Hon and J. M. Arnold, "Stable FDTD on disjoint domains - A discrete Green's function diakoptics approach", The Second European Conference on Antennas and Propagation, EuCAP 2007, pp. 1-6, 2007.

[2] B. P. de Hon and J. M. Arnold, "Stable selfconsistent Green's function diakoptics without spurious recurrences", International Conference on Electromagnetics and Advanced Applications, ICEAA 2011, pp. 1048-1051, 2011.

[3] R. Holtzman and R. Kastner, "The Time-Domain Discrete Green's Function Method (GFM) Characterizing the FDTD Grid Boundary", IEEE Trans. Antennas Propag., vol. 49, no. 7, pp. 107993, 2001.

[4] B. P. de Hon and J. M. Arnold, "Discrete Green's function diakoptics - a toy problem", International Conference on Electromagnetics and Advanced Applications, ICEAA 2010, pp. 565-568, 2010.

[5] B. P. de Hon and J. M. Arnold, "Discrete Green's function diakoptics for stable FDTD interaction between multiply-connected domains", International Conference on Electromagnetics and Advanced Applications, ICEAA 2007, pp. 684-687, 2007. 